\title{
Risk factors for repeated emergency compulsory psychiatric admissions
}

\author{
Mark H. de Jong, André I. Wierdsma, Jeroen Zoeteman, Christina A. van Boeijen, Arthur R. Van Gool and \\ Cornelis L. Mulder
}

\section{Background}

The characteristics of patients who have repeated compulsory psychiatric admissions are largely unknown.

\section{Aims}

To investigate the frequency and risk factors for repeated emergency compulsory psychiatric admission (ECPA); and to identify targets for interventions to reduce repeated ECPA.

\section{Method}

Data were collected from a database of electronic patient files (EPFs) held by three psychiatric emergency services (PES) in the Netherlands. Analyses were based on the data for adult patients (aged 18-75 years) with a first PES contact in 2010-2015. Using descriptive statistics and regression analysis, we studied the associations between baseline patient factors and repeated ECPA and time to readmission, within a 2-year follow-up period.

\section{Results}

We included 6059 patients: $15.6 \%$ had two or more ECPAs. In total, $66 \%$ of second ECPAs had occurred within 6 months of the first. About $30 \%$ of all ECPAs were repeated ECPAs. Two baseline factors were associated with a higher frequency of a second ECPA: history of receiving any mental healthcare treatment, whether in-patient or out-patient or both, and a lower level of self-care. Three were associated with a lower frequency: ethnicity (other than Dutch), older age and suicidality. Lower Global Assessment of Functioning (GAF) scores and housing problems were associated with a shorter time to compulsory readmission and persistent psychiatric problems with a longer time to compulsory readmission.

\section{Conclusions}

We found that $15.6 \%$ of patients had two or more ECPAs. Twothirds of the second ECPAs had occurred within 6 months of the first. Like earlier studies, the risk factors we identified suggest that interventions to reduce the risk of repeated compulsory psychiatric admission should seek to improve self-care, general daily functioning and homelessness.

\section{Keywords}

Emergency psychiatry; compulsory admission; coercion; selfneglect; functioning.

\section{Copyright and usage}

(c) The Author(s), 2020. Published by Cambridge University Press on behalf of the Royal College of Psychiatrists. This is an Open Access article, distributed under the terms of the Creative commons Attribution licence (http://creativecommons.org/ licenses/by/4.0/), which permits unrestricted re-use, distribution, and reproduction in any medium, provided the original work is properly cited.

\section{Background}

Compulsory psychiatric admissions are very stressful events in the lives of patients and their caregivers. ${ }^{1}$ A recent systematic review stated that the greatest risks for such admissions were associated with previous involuntary admissions to hospital and a diagnosis of a psychotic disorder, and to a lesser extent with being male, single and unemployed, receiving welfare benefits and having a diagnosis of bipolar disorder. ${ }^{2}$ The unfortunate fact that many patients undergo repeated compulsory admissions, is indicative of a need to develop targeted interventions.

Most of the little research that has been done on 'revolving-door' patients has focused on voluntary admissions. ${ }^{3-7}$ It found four predictors of frequent readmissions: a history of previous psychiatric admissions, a diagnosis on the psychosis spectrum, being unemployed and living in residential accommodation. But few studies have investigated the prevalence of repeated compulsory psychiatric admissions and their risk factors. During a 5-year follow-up period, a Dutch study found that the frequency of such admissions was $37 \%{ }^{8}$ Compulsory readmission was associated with greater consumption of care in the 5 years before inclusion, a history of compulsory psychiatric admissions, younger age and living alone. During a 7-year follow-up period, a retrospective Taiwanese study in patients with schizophrenia found a frequency of repeated compulsory psychiatric admissions of 5.5\%, and that the risk of compulsory readmission was higher in patients with a prior compulsory admission than in those whose prior admission had been voluntary (adjusted hazard ratio $(\mathrm{HR})=1.31){ }^{9}$ Finally, a prospective study in Switzerland with a 24-month follow-up period found a frequency of repeated compulsory psychiatric admissions of $36 \%$ and that two factors were associated with such admissions: previous compulsory admission - especially when it had been because of endangerment of others - and a diagnosis on the psychosis spectrum. ${ }^{10}$

One important reason for performing these studies is to identify risk factors that may be modifiable targets for interventions intended to reduce repeated compulsory psychiatric admissions. Two of the studies referred to above identified two such risk factors in two different countries: living alone and endangerment of others. As it might be possible to relate these findings to regional variations, it is important to investigate whether they can be replicated in another country.

\section{Purpose of the study}

In our study we therefore focused on identifying targets for interventions intended to reduce repeated emergency compulsory psychiatric admissions (ECPA). Our specific aims were to investigate the following: the frequency of repeated ECPAs; the associations between clinical, demographic and process factors and the risk of repeated ECPA; and the associations between these same factors and the time to compulsory readmission.

\section{Method}

\section{Setting}

Our research included patients using psychiatric emergency services (PES) in three Dutch cities: Amsterdam, Apeldoorn and Rotterdam. 
Whereas Amsterdam and Rotterdam are large cities with extensive suburbs, Apeldoorn is relatively small and has rural surroundings. All three PES are part of an integrated mental healthcare institution in their particular geographic areas. Patients are usually referred by general practitioners, police or physicians in the general hospital.

The three PES involved in the study assessed the patients wherever they were: at home, in the emergency room at a general hospital, at the police station, in the offices of the mental health services or sometimes in an emergency room specially intended for psychiatric crisis care. The crisis assessments were made by a psychiatrist (or a psychiatry resident) and a nurse. After the assessment, the patients were transferred for admission - voluntary or compulsory - or out-patient follow-up or they received no aftercare. To promote the standardisation and uniform registration of all psychiatric crises, they used a concise web-based electronic patient file (EPF) specially designed for acute psychiatric care. Our study is based on the data from these EPFs.

\section{Data and materials}

The EPF data were available from the inception of the database in 2008 up to the end of 2017. Initial inspection of the data showed that the vast majority of second ECPAs had occurred within 2 years of the index ECPA. To create corresponding baseline positions and follow-up periods for all patients included in the analysis, we chose to include unique patients who had no emergency contact in the first 2 years of the database (2008 and 2009) and could be followed for 2 years. We therefore based all our analyses on data from patients whose first PES contact had taken place between 2010 and the end of 2015.

Completing EPFs was part of the daily routine at the three PES. Entries included the patients' demographic and clinical characteristics, such as gender, age, living situation, homelessness, diagnostic category, substance misuse, Global Assessment of Functioning score $(\mathrm{GAF})^{11}$ and the Severity of Psychiatric Illness (SPI) rating scale. ${ }^{12}$ The latter is an instrument for exploring the patient's problems in terms of (a) problem severity; (b) psychiatric complications and comorbidity; and (c) complications in the treatment process. Each item is scored on a four-point scale, ranging from no risk or symptoms, through slight and moderate symptoms to high risk or severe symptoms. The Appendix shows the items that are included on the SPI.

The EPF is also used to register basic process information, including the time at which the contact with the patient took place, the referrer and the type of intervention, i.e. voluntary or compulsory admission, or no admission. To ensure acceptable accuracy with regard to preventing double registrations of the same patient, all patient data were carefully anonymised and a unique EPF identification number was attributed.

The authors assert that all procedures contributing to this work comply with the ethical standards of the relevant national and institutional committees on human experimentation and with the Helsinki Declaration of 1975, as revised in 2008. All procedures involving patients were approved by the Medical Ethics Committee at the Erasmus University Medical Centre (approval number: MEC-2020-0441). Under Dutch legislation on healthcare in general and medical research with human patients in particular, patient file research does not require informed consent. The abovementioned committee classified our study as lying outside the scope of the Netherlands' Medical Research Involving Human Subjects Act, and confirmed that if the patient data was anonymised no informed consent would be required.

\section{Judicial aspects of compulsory psychiatric admissions}

In the Netherlands, ECPA are intended for crises and acutely dangerous situations that require immediate admission (i.e. within $24 \mathrm{~h}$ ).
This procedure requires a mandatory psychiatric assessment by a psychiatrist. According to the legal requirements of an ECPA, a psychiatrist completes the standardised Mental Health Act form (also applicable during the research period) to report on psychiatric status and diagnosis, dangerousness, absence of consent for treatment and admission, and absence of any less radical - i.e. non-compulsory intervention to avert the present danger. After completion of the mandatory procedure, the detention is sanctioned by the mayor. Within 5 working days, a judge reviews the mayor's decision in a court session in the psychiatric clinic.

\section{Statistical analysis}

Descriptive statistics were used to summarise the demographic, clinical and contextual characteristics of all patients who had either a single ECPA or two or more ECPAs. Multiple logistic regression analysis was used to identify variables associated with repeated ECPA, and Cox regression analysis to explore variations in the time to compulsory readmission. As well as patient characteristics and process factors, each SPI item was separately entered into the model. Following Hosmer \& Lemeshow, variable selection was based on a stepwise procedure with $P<0.20$ as entry level and $P>0.05$ as removal level. ${ }^{13}$ Odds ratio (OR) estimates and their corresponding $95 \%$ CIs were calculated for explanatory variables in the final models. All statistical analyses were performed using SPSS version 25 .

\section{Results}

\section{Patients}

From 2010 through 2015, the three PES assessed a total of 27186 patients (aged 18-75 years), 6059 of whom (22.3\%) had one or more ECPAs. Table 1 summarises the patient characteristics of patients who had one ECPA and of those who had two or more ECPAs.

\section{Frequency of ECPAs}

In the group of 6059 patients who had been admitted compulsorily between 2010 and the end of 2015, 945 patients (15.6\%) had two or more ECPAs within 2 years of the index ECPA. These 945 patients counted for nearly $30 \%$ of the total number of ECPAs during the observation period, $12.3 \%$ of them having had two ECPAs

Table 1 Baseline characteristics of all patients with one emergency compulsory psychiatric admission (ECPA) and with two or more ECPAs (first ECPA 2010-2015)

\begin{tabular}{|c|c|c|c|}
\hline & 1 ECPA & $\geq 2$ ECPAS & Total \\
\hline$n$ & 5114 & 945 & 6059 \\
\hline Gender, \% male & 61.0 & 62.0 & 61.1 \\
\hline Age, years: median (IR) & $40(23)$ & $37(23)$ & $39(23)$ \\
\hline Ethnicity, \% born Dutch ${ }^{a}$ & 76.0 & 79.7 & 76.6 \\
\hline Living with family, \% ${ }^{\mathrm{b}}$ & 36.1 & 38.8 & 36.6 \\
\hline Homeless, \% & 6.5 & 6.5 & 6.5 \\
\hline History of any mental healthcare, \% & 29.2 & 32.5 & 29.7 \\
\hline $\begin{array}{l}\text { Psychotic disorder, primary } \\
\text { diagnosis \% }\end{array}$ & 60.5 & 61.4 & 60.7 \\
\hline $\begin{array}{l}\text { Personality disorder, primary } \\
\quad \text { diagnosis \% }\end{array}$ & 2.1 & 3.5 & 2.3 \\
\hline Any alcohol or drugs misuse, $\%^{c}$ & 62.6 & 65.5 & 63.0 \\
\hline SPI sum, mean (s.d.) & $25.6(5.6)$ & $25.8(5.3)$ & $25.7(5.6)$ \\
\hline GAF score, mean (s.d.) & $30.4(9.2)$ & $30.2(9.0)$ & $30.4(9.2)$ \\
\hline \multicolumn{4}{|c|}{$\begin{array}{l}\text { IR, interquartile range; SPI, Severity of Psychiatric IIIness; GAF, Global Assessment of } \\
\text { Functioning. } \\
\text { a. Data available for } n=4755 \text {. } \\
\text { b. Data available for } n=4652 \text {. } \\
\text { c. Data available for } n=4190 \text {. }\end{array}$} \\
\hline
\end{tabular}


(accounting for $20.5 \%$ of the total number of ECPAs); $2.4 \%$ having had three ECPAs $(6.1 \%$ of the total); and $0.8 \%$ having had four ECPAs $(2.9 \%$ of the total). Two-thirds of all second ECPAs (66.1\%) had occurred within 6 months of the first.

The small but interesting subgroup of patients who had $\geq 4$ ECPAs during the observation period $(n=53)$ - who, unsurprisingly, represented the largest proportion of readmissions within 6 months $(81.1 \%)$ - had several specific characteristics, representing the largest proportion of patients with a baseline history of receiving any psychiatric treatment, whether in-patient or out-patient or both $(34.0 \%, n=18)$, with a severe score on the SPI item suicidality $(32.1 \%, n=17)$, with a personality disorder $(7.5 \%, n=4)$, with alcohol or drugs misuse $(71.8 \%, n=38)$ and of those who were homeless $(15.1 \%, n=8)$.

\section{Factors associated with compulsory readmission}

The following baseline factors were associated with a higher frequency of repeated ECPAs: history of receiving any psychiatric treatment, whether in-patient, or out-patient, or both $(\mathrm{OR})=1.23,95 \% \mathrm{CI} 1.05-1.44)$ and lower level of self-care $(\mathrm{OR}=$ $1.21,95 \%$ CI 1.03-1.42). Baseline factors associated with a lower frequency of repeated ECPAs were ethnicity (other than Dutch) $(\mathrm{OR}=$ $0.71,95 \%$ CI 0.59-0.85); older age ( $\mathrm{OR}=0.98,95 \%$ CI 0.97-0.99); and suicidality $(\mathrm{OR}=0.76,95 \% \mathrm{CI} 0.65-0.90)$.

\section{Regional variations}

Analysis of the details of the three PES regions showed that a history of receiving any psychiatric treatment, whether in-patient, or outpatient, or both was an important factor in the risk of compulsory readmission, but to a slightly lesser extent in the rural area than in urban areas. Whereas ethnicity was relevant specifically in Amsterdam, age was of particular importance in Rotterdam, and level of self-care in Apeldoorn. A subanalysis showed that, independently of patient and process characteristics, PES region was associated with the risk of repeated ECPA. Details are available on request from the authors.

\section{Factors associated with time to readmission}

The Cox regression model showed that lower GAF scores and housing problems were associated with shorter time to compulsory readmission $(\mathrm{HR}=1.01,95 \% \mathrm{CI} 1.00-1.02$ and $\mathrm{HR}=1.17,95 \% \mathrm{CI}$ $1.02-1.34$, respectively). It also showed that persistent psychiatric problems were associated with a longer time to compulsory readmission $(\mathrm{HR}=0.85,95 \% \mathrm{CI} 0.73-1.00)$.

\section{Discussion}

\section{Main results}

In this investigation of the frequency and risk factors for repeated ECPA, we found that $15.6 \%$ of patients had two or more ECPAs. Approximately $30 \%$ of all ECPAs were repeated ECPAs. The literature reports considerable variations in the frequencies of repeated compulsory psychiatric admission: $5.5 \%$ in 7 years (Taiwan); ${ }^{9} 36 \%$ in 2 years (Switzerland); ${ }^{10}$ and $37 \%$ in 5 years (the Netherlands). ${ }^{8}$ Our own finding of $15.6 \%$ over 2 years takes account of the different lengths of follow-up, and expresses an average.

We also found that two-thirds of second ECPAs (66.1\%) occurred within 6 months of the first. This is similar first to the findings of Lay et al, who reported a peak of compulsory readmissions shortly after discharge; ${ }^{10}$ and also to the findings of van der Post et al, who reported a higher risk of readmission in the first year after discharge than in the subsequent follow-up years. ${ }^{8}$ However, in the first year of follow-up, the study by Lin et al showed relatively low rates of compulsory readmissions. ${ }^{9}$

As well as three static, unmodifiable baseline risk factors for a repeated ECPA - history of receiving any psychiatric treatment whether in-patient, or out-patient, or both, age and ethnicity - we found one relevant factor that could potentially be modified by interventions: level of self-care. With regard to the time to readmission lower GAF scores and housing problems were also potentially modifiable risk factors.

Our findings with regard to history of mental healthcare and age are similar to those in the other studies, in which younger age and an existing treatment history in mental healthcare were consistently identified as important risk factors for repeated ECPAs. ${ }^{8-10}$ Our findings with regard to various factors - level of self-care, housing problems and lower GAF scores - also indicate that patients with severe mental illnesses ${ }^{14,15}$ who show lower level of functioning are particularly susceptible to repeated compulsory psychiatric admissions. This, too, is in accordance with the literature on revolving-door patients. ${ }^{3-7}$

Our finding with regard to ethnicity requires some deliberation. Although one would expect ethnicity other than that of the majority group to be associated with a higher risk of repeated ECPA, ${ }^{16}$ it was clear from the regional differences that ethnicity was important mainly in Amsterdam. This association almost certainly lies in the millions of foreign tourists who visited this city in the period in question. As a result of various psychiatric crises, some of them required the services of the PES and were compulsorily admitted. Once they had returned to their own country, the chance of a repeated ECPA in Amsterdam was close to zero. The effect of these large numbers of short-term foreign visitors probably exceeded any differences in the proportions of Amsterdam's native Dutch residents and its other long-term residents.

\section{Strengths and limitations}

To our knowledge, this study is one of the few to have investigated risk factors for repeated compulsory psychiatric admissions. The database, on which our study was based, had various strengths in its own right. First, it contained a large number of patients and individual PES contacts, and reflected routine daily practice. Second, the EPF was standardised, included a structured assessment scale, and was concise and easy to use. A further strength of the study was the absence of inclusion and exclusion criteria, which provided an advantage with regard to representativeness and generalisability.

There are also some limitations. There were relatively large proportions of missing data on a few database items, such as ethnicity, living situation and substance misuse. A further limitation lay in an effect of our explorative approach, thus, findings do not identify causal factors and relationships but associations - merely suggesting the direction of further research. Also, the clinical data with regard to the compulsory hospital stay and other follow-up treatment after assessment by the PES was not accessible for this study. For example, we were unable to incorporate data on psychotherapeutic and psychopharmacological interventions and determine their relationship with repeated ECPAs. Finally, the odds and hazard ratios were generally relatively small.

\section{Clinical implications}

The importance of preventive measures is demonstrated by the large numbers of patients with an ECPA - and particularly of those with repeated ECPAs - that were found in our study and in earlier studies. On the basis both of earlier findings and of the possible risk factors we identified, interventions to reduce the risk of repeated compulsory psychiatric admissions should focus on improving self-care, general daily functioning and housing 
problems. To reduce the risk of repeated ECPAs, future studies might then investigate the effects of interventions that specifically target these factors. Examples of these interventions could be found in integrated out-patient treatment programmes, such as flexible assertive community therapy, ${ }^{17}$ which not only focuses on psychopathology but also pays attention to patients' daily functioning, activities, social network and housing.

Finally, two-thirds of the second ECPAs in our study occurred within 6 months - a very short period. This finding suggests that regardless of their demographic and clinical characteristics, any patient with a recent or very recent ECPA deserves intensive follow-up, and that this should focus on reducing the risk of repeated compulsory psychiatric admission.

Mark H. de Jong $(\mathbb{D}, \mathrm{MD}$, Yulius Mental Health, the Netherlands; André I. Wierdsma PhD, Epidemiological and Social Psychiatric Research Institute (ESPRi), Department of Psychiatry, Erasmus University Medical Centre, the Netherlands; Jeroen Zoeteman, MD, Spoedeisende Psychiatrie Amsterdam, the Netherlands; Christina A. van Boeijen, MD, PhD, GGNet, the Netherlands; Arthur R. Van Gool, MD, PhD, Emergis, the Netherlands; Cornelis L. Mulder, MD, PhD, Epidemiological and Social Psychiatric Research Institute (ESPRi), Department of Psychiatry, Erasmus University Medical Centre, the Netherlands

Correspondence: Mark H. de Jong. Email: ma.dejong@yulius.nl

First received 4 Sep 2020, final revision 24 Oct 2020, accepted 23 Nov 2020

\section{Data availability}

The data that support the findings of this study are available from the corresponding author, M.H.d.J., upon reasonable request.

\section{Author contributions}

M.H.d.J. designed the work, analysed and interpreted the data, drafted the manuscript, under took critical revision of the manuscript, gave final approval of the manuscript, and agreed to be accountable for all aspects of the work. A.I.W. designed the work, analysed and interpreted the data, undertook critical revision of the manuscript, gave final approval of the manuscript, and agreed to be accountable for all aspects of the work. J.z. designed the work, undertook critical revision of the manuscript, gave final approval of the manuscript, and agreed to be accountable for all aspects of the work. C.A.v.B. designed the work, undertook critical revision of the manuscript, gave final approval of the manuscript, and agreed to be accountable for all aspects of the work. A.R.V.G. designed the work, analysed and interpreted the data, undertook critical revision of the manuscript, gave final approval of the manuscript, and agreed to be accountable for all aspects of the work. C.L.M. designed the work, analysed and interpreted the data, undertook critical revision of the manuscript, gave final approval of the manuscript, and agreed to be accountable for all aspects of the work.

\section{Declaration of interest}

None.

\section{Appendix}

\section{Items on the Severity of Psychiatric Illness rating scale}

Item

Severity of the problems

suicide potential

Danger to others

Severity of psychiatric symptoms

Level of self-care

Psychiatric complications and comorbidity
5 Substance abuse

6 Medical complications

7 Family problems

8 Vocational problems

Complications in the treatment process

Housing problems

10 Motivation for treatment

11 Medication compliance

12 Illness insight

13 Family involvement

14 Symptom persistence

\section{References}

1 Frueh BC, Knapp RG, Cusack KJ, Grubaugh AL, Sauvageot JA, Cousins VC, et al. Patients' reports of traumatic or harmful experiences within the psychiatric setting. Psychiatr Serv 2005; 56: 1123-33.

2 Walker S, Mackay E, Barnett P, Sheridan Rains L, Leverton M, Dalton-Locke C, et al. Clinical and social factors associated with increased risk for involuntary psychiatric hospitalisation: a systematic review, meta-analysis, and narrative synthesis. Lancet Psychiatry 2019; 6: 1039-53.

3 Schmutte T, Dunn CL, Sledge WH. Predicting time to readmission in patients with recent histories of recurrent psychiatric hospitalization: a matched-control survival analysis. J Nerv Ment Dis 2010; 198: 860-3.

4 Evans LJ, Harris V, Newman L, Beck A. Rapid and frequent psychiatric readmissions: associated factors. Int J Psychiatry Clin Pract 2017; 21: 271-6.

5 Di Lorenzo R, Sagona M, Landi G, Martire L, Piemonte C, Del Giovane C. The revolving door phenomenon in an Italian acute psychiatric ward: a 5-year retrospective analysis of the potential risk factors. J Nerv Ment Dis 2016; 204: 686-92.

6 Lewis T, Joyce PR. The new revolving-door patients: results from a national cohort of first admissions. Acta Psychiatr Scand 1990; 82: 130-5.

7 Woogh CM. A cohort through the revolving door. Can J Psychiatry 1986; 31: 214-21.

8 van der Post LFM, Beekman ATF, Peen J, Zoeteman J, Twisk JWR, Dekker JJM. Patterns of care consumption after compulsory admission: a five-year followup to the Amsterdam study of acute psychiatry VIII. Int J Ment Health 2016; 45: 105-17.

9 Lin CE, Chung CH, Chen LF, Chen PC, Cheng HY, Chien WC. Compulsory admission is associated with an increased risk of readmission in patients with schizophrenia: a 7-year, population-based, retrospective cohort study. Soc Psychiatry Psychiatr Epidemiol 2019; 54: 243-53.

10 Lay B, Kawohl W, Rossler W. Predictors of compulsory re-admission to psychiatric inpatient care. Front Psychiatry 2019; 10: 120.

11 Startup M, Jackson MC, Bendix S. The concurrent validity of the Global Assessment of Functioning (GAF). Br J Clin Psychol 2002; 41: 417-22.

12 Lyons JS, Colletta J, Devens M, Finkel SI. Validity of the Severity of Psychiatric Illness rating scale in a sample of inpatients on a psychogeriatric unit. Int Psychogeriatr 1995; 7: 407-16.

13 Hosmer DW, Lemeshow S. Applied Logistic Regression (2nd edn). Wiley, 2000.

14 Ruggeri M, Leese M, Thornicroft G, Bisoffi G, Tansella M. Definition and prevalence of severe and persistent mental illness. Br J Psychiatry 2000; 177: 149-55.

15 Delespaul $\mathrm{PH}$, de consensusgroep EPA. Consensus regarding the definition of persons with severe mental illness and the number of such persons in the Netherlands [in Dutch]. Tijdschr Psychiatr 2013; 55: 427-38.

16 Barnett P, Mackay E, Matthews H, Gate R, Greenwood H, Ariyo K, et al. Ethnic variations in compulsory detention under the Mental Health Act: a systematic review and meta-analysis of international data. Lancet Psychiatry 2019; 6: 305-17.

17 van Veldhuizen JR. FACT: a Dutch version of ACT. Community Ment Health J 2007; 43: 421-33 